Background Disregulated apoptosis of T lymphocytes was postulated as one of the potential patomechanisms of rheumatoid arthritis. In peripheral blood of RA patients atypical clones of $\mathrm{T}$ cells were identified, overexpressing Bcl-2 protein and apoptosis resistant. Experimental treatment inducing apoptosis of joint infiltrating $\mathrm{T}$ cells and synovial fibroblasts resulted in disease remission in animal models.

Objectives The objective of the study was to examine the expression of apoptosis regulatory proteins ( $\mathrm{p} 53, \mathrm{Bcl}-2$, Bax and Fas) in peripheral blood and synovial fluid lymphocytes as well as sFas concentrations in patients with RA and to compare it with expression in other arthritides. We analized the correlation with disease clinical characteristics and treatment. The other aim was to determine the relation of those markers expression in blood and synovial fluid lymphocytes from the same patients.

Methods Study covered 77 RA patients, 18 OA patients 10 patients with other arthritides and healthy control group. Immunocytochemical analysis was performed with indirect immunoperoxidase test. sFas concentration was measured with ELISA test.

Results In RA patients with advanced joint destruction and active disease high overexpression of $\mathrm{Bcl}-2$ was found (over $80 \%$ of cells) which correlated to ESR $(r=0.63, p=0.027)$. Bcl-2 expression was significantly higher in RA patients with limited disease than in pts with extra articular disease $(p=0.02)$ No significant difference in other apoptosis markers expression among examined groups was found. We did not observe its relation to disease duration, RF seropositivity, clinical stage or treatment. Serum sFas concentration was significantly higher in extra articular RA ( $\mathrm{p}=0.009)$.

Conclusion The above results may indirectly suggest the role of lymphocyte apoptosis defect in RA pathogenesis, which demands further investigation.

\section{THU0124 AUTOLOGOUS FIBROBLASTS AS VECTORS FOR ANTI_INFLAMMATORY GENE THERAPY IN MURINE COLLAGEN-INDUCED ARTHRITIS}

N Bessis, V Cottard, N Saidenberg-Kermanach, MC Boissier. Immunology, UFR Léonard de Vinci, UPRES EA-2361, Bobigny, France

10.1136/annrheumdis-2001.1001

Background No treatments effective in the long term are available for rheumatoid arthritis.

Objectives Recent advances in gene therapy and cell therapy have prompted us to design for collagen-induced arthritis (CIA), a new treatment strategy involving injection of autologous fibroblasts transfected with the IL-4 gene using a nonviral method.

Methods A line of immortalised fibroblasts from DBA/1 mice (DBA/1/0 cells) were transfected with a plasmid expressing IL-4 cDNA (DBA/1/IL-4 cells). Xenogeneic fibroblasts from Chinese hamster ovary $(\mathrm{CHO})$ transfected with a plasmid expressing murine IL-4 cDNA were studied also. CIA was caused in DBA/1 mice by injecting bovine type II collagen (CIIB). The cells were injected into the subcutaneous tissue of the back 10 and 25 days after immunisation of the mice by $3.106 \mathrm{DBA} / 1 / 0$ or DBA/1/IL4 or $\mathrm{CHO} / \mathrm{IL}-4$ cells.

Results Injection of DBA/1/IL-4 cells was associated with a significant and lasting improvement in the clinical and histological evidence of joint inflammation and destruction as compared with DBA/ $1 / 0$ cells and CHO/IL- 4 cells. The mice injected with DBA/ $1 / \mathrm{IL}-4$ cells showed decreases in the production of $\operatorname{IgG} 2 \mathrm{a}$ antibody to CII and in the proliferation of CIIB-specific nodal $\mathrm{T}$ cells.

Conclusion Taken in aggregate, these findings indicate that grafting autologous cells transfected with a gene encoding an antiinflammatory cytokine is effective in CIA and may attenuate the cytokine imbalance seen in this disease.

Rheumatoid Arthritis - Clinical aspects

\section{AB0037 COMPLICATED RA COURSE WITH ONSET IN ELDERLY AGE}

AM Satybaldyev, TF Akimova, MM Ivanova. Laboratory of Prognosis and Outcomes of Rheumatic Diseases, Institute of Rheumatology of RAMS, Moscow, Russia

10.1136/annrheumdis-2001.1002

\section{Background}

\section{Objectives}

Methods Disease course in 173 RA pts with onset in age older than 50 was analysed. It was demonstrated that in 101 pts (M:F $=12: 89$, median onset $58.6 \mathrm{y} / \mathrm{o}$, median disease duration up to complication - 6.9 yrs, median age of last examination 67.5, median observation period 7.5) the following complications developed: aseptic bone necrosis, secondary amyloidosis, expressed osteoporosis with osteoporotic bone fractures and compression of vertebral bodies.

Results Systemic manifestations (rheumatoid nodes, internal organs lesions, symptoms of serositis) were determined in $36 \%$ of pts, rheumatoid factor in 92\%. Vasculopathies including livedo reticularis, capillarites and trophic ulcers - in 20\% of pts.

Aseptic bone necrosis was found in 40 pts (median observation period $10.2 \mathrm{yrs}$ ). Their localization: femoral head and/or tectum of cotyloid cavity in 20 pts, subchondral bones of knee joint - in 14, less frequently in wrist, humerus and elbow joints. Repeated aseptic necroses developed in 31 pts.

31 pts had secondary amyloidosis, median disease duration up to diagnosis - 7.6 yrs. This group of pts demonstrated highest inflammatory activity according to clinical and laboratory data. Observation period of pts with already developed amyloidosis was from 2 to 10.5 yrs. 14 pts out of them died during the period of observation.

Developed osteoporosis and compression of vertebrae were observed in 35 pts (median age of fractures 63.8). Radiodensitometry in all pts demonstrated reduced mineral bone density more than $2.5 \mathrm{CD}$. Localization of skeletal bones fractures (in 24 pts) was as follows: femoral head in $35 \%$ of cases, ribs - in $20 \%$, humerus - in $15 \%$, less frequently radius, pelvic bones, feet. Multiple fractures were observed in $40 \%$ of cases.

Compressions of vertebral bodies were found in 19 pts, combinations of osteoporotic fractures and vertebral bodies compression - in 9 pts.

Conclusion Thus, follow-up of elderly pts enabled us to select the group of pts with complicated disease course aggravated the prognosis. Reducing of high inflammatory activity of the disease by active therapy will be positive for the disease course, decrease the risk of complications associated with amyloidosis, with diseases activity, aseptic bone necrosis, osteoporosis.

Preventive measures are recommended to decrease osteoporotic complications from the very beginning of the disease. 